A. G. Biney and H. Sellahewa, "Analysis of smartphone model identification using digital images," presented at the 20th IEEE International Conference on Image Processing, Melbourne, Australia, 2015.

(C) 2015 IEEE. Personal use of this material is permitted. Permission from IEEE must be obtained for all other uses, in any current or future media, including reprinting/republishing this material for advertising or promotional purposes, creating new collective works, for resale or redistribution to servers or lists, or reuse of any copyrighted component of this work in other works. 


\title{
ANALYSIS OF SMARTPHONE MODEL IDENTIFICATION USING DIGITAL IMAGES
}

\author{
Akua G. Biney and Harin Sellahewa \\ Department of Applied Computing \\ University of Buckingham \\ Buckingham, UK
}

\begin{abstract}
This paper is focused on smartphone model identification using image features. A total of 64 image features - broadly categorized into colour features, wavelet features and image quality features - are extracted from high-resolution smartphone images. A binary-class turned to multiclass support vector machine (SVM) is used as the classifier. Experimental results based on 1800 images captured with 10 different smartphone/tablet devices are promising in correctly identifying source smartphone model. Image quality metrics and wavelet features are shown to contain the most useful device/model information compared to colour features. However, compared to colour features, quality and wavelet features are highly sensitive to simple image modifications. The combined set of colour, quality and wavelet features achieves the overall best identification accuracy.
\end{abstract}

Index Terms - Smartphone Identification, Forensics, Image Features, Wavelet Transforms, Support Vector Machine

\section{INTRODUCTION}

The rising need to prove the originality and authenticity of digital images and investigate crimes committed using digital images such as child pornography, blackmail and bullying, have led to various research and studies in to ways of identifying source devices of digital images. Identifying the source device of an image or a video in question could provide vital evidence to forensic investigators in solving a case. Many identification methods and approaches ranging from extracting information from the JPEG or EXIF header, image histogram based methods [1], JPEG compression methods [2], and image feature based methods [3, 4], among others have been proposed to identify source devices of digital images.

Most images nowadays, are captured using smartphones and tablets because they are highly portable, mobile, affordable, and they consist of high-quality cameras and a variety of connectivity options that enable the user to instantly share images with others. Statistics show that more people

The first author would like to thank the Commonwealth Shared Scholarship Scheme for funding her master degree. own mobile phones than digital cameras and sales of smartphones continue to increase. Crimes involving images and videos captured by smartphones have been long recognised by the law with the increase in availability of mobile devices coupled with the devices' capability for various activities like web browsing, online social networking, email, MMS, video chat, etc. Previous research on source device identification using digital images focuses more on typical digital cameras, printers, scanners and other computer aided or generated images. However, not so much has been done with respect to identifying cameras embedded in smartphones or the smartphones themselves.

The purpose of this paper is to use and evaluate existing state-of-the-art techniques to develop a model to further help improve work being done in the field of source model/device identification. In particular, we investigate the use of image features to identify the smartphone model used to capture a given unknown image.

In related works, Kharrazi et al. [5] proposed the use of colour, wavelet and image quality metrics features - a total of 34 features - for blind source camera identification; a Support Vector Machine (SVM) classifier is used to identify cell phones.. Celiktutan et al. [6] used an extended set of $592 \mathrm{im}$ age features that included binary similarity measures, image quality measures, and higher order wavelet statistics. Feature selection algorithms were used to select the optimal set of features at feature-level fusion. Moreover, a decision-level fusion was also considered to classify 16 cell phone models. In similar work, Tsai et al. [7] used colour, quality, and frequency domain image characteristics (obtained using Haar wavelet) for camera/mobile phone source identification.

More recently, Gloe et al. used these features to train and classify 9185 images from 11 digital camera devices, resulting in a $97.79 \%$ accuracy [8]. Following up on their previous study, Gloe and Böhme presented a much larger image database - Dresden Image Database - to benchmark camerabased digital image forensic techniques [9]. In [10], Van et al. worked on identifying cell phone source devices using only lateral chromatic aberration features.

This paper uses the image feature-based approach presented by Kharrazi et al. [5] to identify the source smartphone model of a given image. Our contributions are: a) 
we evaluate the performance of each image feature category on its own as well as taking them as a combined feature set; b) we investigated the effects of image cropping and resizing on smartphone model identification accuracy; c) we designed a new smartphone image database to evaluate existing techniques for source model identification. The Smartphone Image Database consists of some of the most widely used modern smartphone brands/models today and the images are captured at a much higher resolution than those found in previous studies. The database, described in section 3 , is available to other researchers - the database can be obtained by contacting the second author - to continue work in this field.

The rest of the paper is organised as follows. Section 2 briefly describes the image features and the classification model trained for source device identification. Image data collection, smartphone devices and different image sizes are explained in section 3. Section 4 presents experimental results and a discussion. Our conclusions and future direction of work are given in section 5 .

\section{IMAGE FEATURES AND CLASSIFICATION}

In our work, we use colour, wavelet and image quality metrics (IQM) features which takes advantage of all the stages in the image acquisition pipeline to identify modern smartphone devices linked to images $[5,6]$. This approach analyses the relationship between a source device and its associated image. It believes that all cameras (typical digital cameras and smartphone cameras) follow the same image acquisition process so the components and configuration settings of these devices which differ from manufacturer to manufacturer - and to some extent considered a trade secret - could be a good approach to use in distinguishing one device from another. The different components and configuration settings used have significant effect on the image produced; hence features from images could be used to trace their source devices $[5,6,9]$.

In the colour image feature category, the mean, variance, neighbour distribution centre of mass and the energy ratios were computed for each image. The denoised image version needed to compute IQM features was obtained by adding Gaussian noise of zero mean and unit variance. A median filter is then used to remove the added noise. The Haar wavelet transform at level one was used to calculate the wavelet features. In our case, we used the sum of coefficients in the three corresponding high-frequency wavelet subbands to extract relevant features and we found that the results of these features were similar to using features extracted from each subband. In essence, we have reduced the total number of image features from 64 to 40 .

The extracted features are fed to a SVM [11] to train a model that would be used to classify new image instances. A binary-class turned to multiclass support vector machine (SVM) is used as the classifier. The final decision on the source model is based on the majority vote count. The SVM non-linear radial basis Function (RBF) kernel with parameter settings of $\mathrm{c}=100$ and default gamma values were used in all experiments. Results were analysed to get distinct features that are significant in classifying images from smartphone devices.

\section{SMARTPHONE IMAGE DATABASE}

The image database used in this study consists of 600 original size - the highest resolution available - images of buildings, sky, roads, fields, rooms, indoor scenes, outdoor scenes and natural environments around the campus. Images were captured using 10 modern smartphones representing 9 different smartphone models - there were two HTC devices of the same model. Each device was used in taking 60 images; in as much as it was possible, the same scenes were captured with all available devices. This actually is not supposed to affect the performance of the classifier, because, the model should be able to correctly predict the source device irrespective of the scene or environment or conditions under which an image is captured. In addition, a set of 600 images were created by resizing the original images to $800 \times 600$ pixels (the resized images of Nokia N8 were $800 \times 450$ pixels) and a further set of 600 images were created by randomly cropping out a $700 \times 700$ region from each original image. These two image sets were created to evaluate the accuracy of smartphone identification using a cropped or resized version of the original image. Thus, our smartphone image database has a total of 1800 images. Few examples of original images are shown in figure 1. Examples of original images along with their cropped version are shown in figure 2.

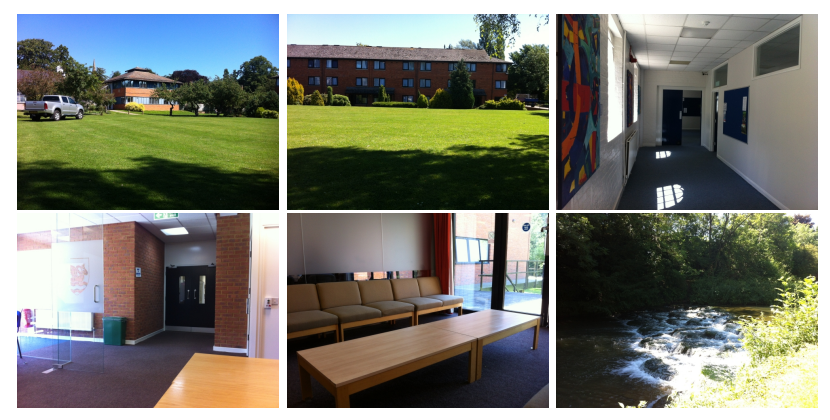

Fig. 1: Example images of the smartphone image database.

\section{EXPERIMENTS}

We conducted a number of smartphone model identification experiments to evaluate the performance of image features and SVM classifier. Firstly, we evaluated the effect of training and testing sample sizes on identification accuracy. Three percentage splits (i.e. $10 \%, 50 \%$, and $90 \%$ images randomly selected for training and the remainder is used for testing) 


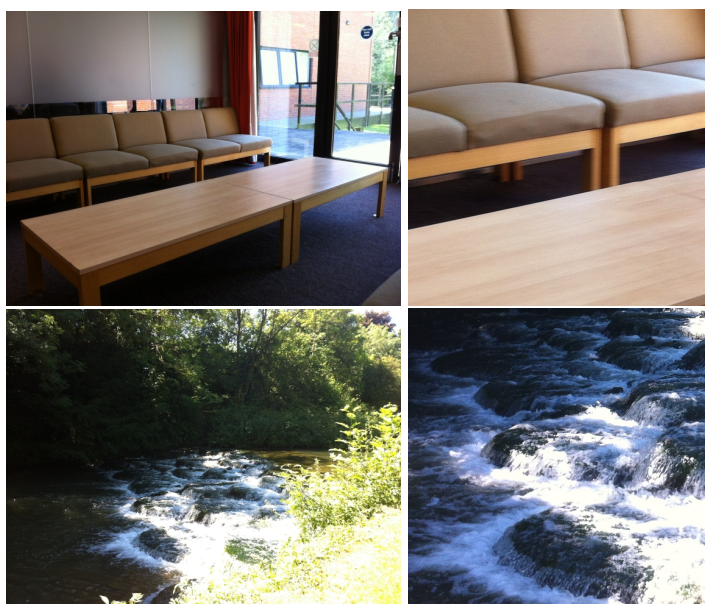

Fig. 2: Original images (left) and cropped images (right) of the smartphone image database.

were used in each experiment. Note that there was no overlap between training and test images. For each sample size, we evaluated the accuracy of colour features, quality features and wavelet features separately as well as in combination as a single feature representation. Furthermore, we evaluated the scenarios where the SVM is trained using original images whilst the test images were either cropped regions of original images or resized versions of original images.

\subsection{Original images}

Experiments on original size images (used for training and testing) showed overall accuracy rates of $62 \%, 64 \%$ and $76 \%$ for using only colour features in 10\%, 50\% and $90 \%$ splits respectively. Using only IQM features, achieved accuracy rates were $77 \%, 88 \%, 96 \%$ respectively. Wavelet features achieved accuracy rates of $82 \%, 88 \%$, and $95 \%$ for the three percentage splits respectively. Using all image features accuracy rates are $80 \%, 90 \%$, and $97.5 \%$, which is an improvement in accuracy over all individual feature types. An $80 \%$ accuracy rate with only 6 images per smartphone model used in training is significant. Table 1 shows overall smartphone model identification results for individual and combined features based on original images. Individual model/device identification accuracy rates are shown in figure 3. It is worth noting that the classifier has been able distinguish between two devices of the same HTC model.

\subsection{Resized images}

Table 2 shows overall smartphone model identification results for individual and combined features based on resized images. Results show an overall identification accuracy of 92\%; a descrease in performance compared to the $97.5 \%$ accuracy achieved on original size images using all features. This attest

\begin{tabular}{c|cccc}
\hline $\begin{array}{c}\text { Training } \\
\text { Data }\end{array}$ & \multicolumn{4}{|c}{ Image Features } \\
\hline $10 \%$ & 62 & 77 & 82 & 80 \\
$50 \%$ & 64 & 88 & 88 & 90 \\
$90 \%$ & 76 & 96 & 95 & 97.5 \\
\hline
\end{tabular}

Table 1: Identification accuracy of individual and combined features using original images in training and testing.

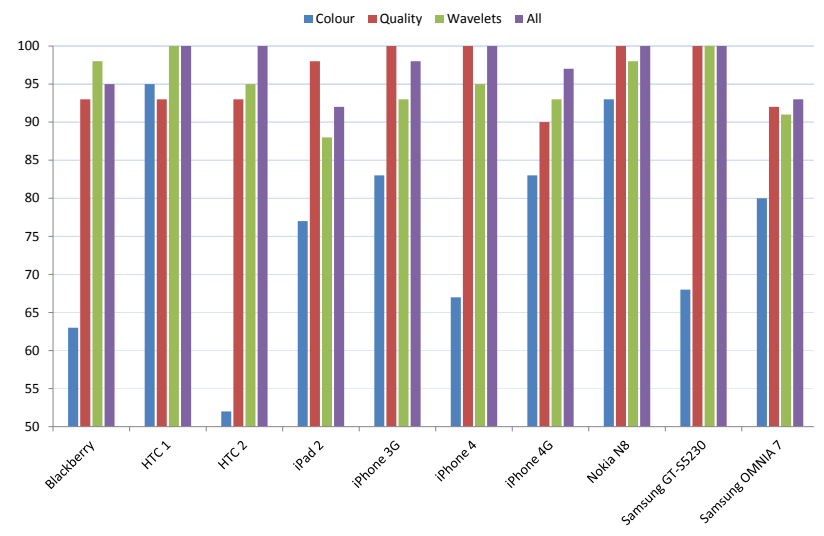

Fig. 3: Identification accuracy of individual devices using original images in training and testing.

to the fact that scaled images, and modified images in general, presents more challenges in image source device/model identification. The $92 \%$ accuracy resulted from combining all image features and using $90 \%$ of the images for training and the remaining $10 \%$ for testing. IQM features were significant in this experiment by achieving the highest accuracy of $85 \%$. Wavelet features and colour features presented nearly the same accuracy of $77 \%$ and $75 \%$ respectively.

Using half of the images in training and the other half in testing, combining all features resulted in $73 \%$ accuracy; IQM features achieved an accuracy of $72 \%$; wavelet features $70 \%$ whilst colour features reached an accuracy level of $65 \%$. Using $10 \%$ of images from each device in training and $90 \%$ in testing, combined features achieved only $63 \%$ accuracy when compared to $80 \%$ for the same scenario with original images. IQM features achieved 65\% accuracy; wavelet features $60 \%$ accuracy; and colour features an accuracy of $62 \%$. The sharp decrease in accuracy for wavelet features can be attributed to the fact that scaled images makes the coefficient of the resulting high frequencies much closer to zero (due to smoothing), therefore increasing similarities across devices/models. Wavelet features are also highly sensitive to distortions caused by resizing an image. The same could explain the significant drop in accuracy with respect to IQM features. Individual device accuracy rates are given in figure 4. 


\begin{tabular}{c|cccc}
\hline $\begin{array}{c}\text { Training } \\
\text { Data }\end{array}$ & \multicolumn{4}{|c}{ Image Features } \\
\hline $10 \%$ & 62 & 65 & 60 & 63 \\
$50 \%$ & 65 & 72 & 70 & 73 \\
$90 \%$ & 75 & 85 & 77 & 92 \\
\hline
\end{tabular}

Table 2: Identification accuracy of individual and combined features using resized images in training and testing.

\subsection{Cropped images}

Similar to results reported on resized images, results on cropped images showed an overall accuracy of $93 \%$ - a significant drop in accuracy when compared to using original images in training and testing. This accuracy resulted from using all features and using $90 \%$ of images in training. Similarly, IQM features and wavelet features were significant in this experiment reporting $85 \%$ and $84 \%$ accuracy respectively. However, these features were the most sensitive to image cropping. Colour features report $71 \%$ accuracy and remained relatively unaffected due to image cropping.

Using 50\% of the images in training, combining all features showed $81 \%$ accuracy, wavelet features presented $78 \%$ accuracy; IQM features reported $76 \%$ accuracy whiles colour features presented $65 \%$ accuracy. When $10 \%$ of the total images were used in training, results for combining all features gave an accuracy of $68 \%$, using only wavelet features presented $71 \%$ accuracy, IQM features and colour features showed rates of $68 \%$ and $61 \%$ respectively.

\begin{tabular}{c|cccc}
\hline $\begin{array}{c}\text { Training } \\
\text { Data }\end{array}$ & \multicolumn{4}{|c}{ Image Features } \\
\hline $10 \%$ & 61 & 68 & 71 & 68 \\
$50 \%$ & 65 & 76 & 78 & 81 \\
$90 \%$ & 71 & 85 & 84 & 93 \\
\hline
\end{tabular}

Table 3: Identification accuracy of individual and combined features using cropped images in training and testing.

\subsection{Mixed images}

A series of experiments were conducted using the different image sizes to train (i.e. original images) and test (i.e. resized and cropped images) the model. This is to further test the robustness of the model against modified images. Individual device/model accuracy rates can be seen in figure 4 .

Using $90 \%$ of original images in training and testing with $90 \%$ cropped images, overall accuracy is $76.5 \%$. Highest individual accuracy of $91.85 \%$ is from the Samsung Omnia 7 device and the lowest being iPhone- 4 with $48.33 \%$. Also, there were some similarities compared with previous results in terms of intra-device similarities. HTC1 lost 5\% to its counterpart, HTC2. Similarly, 5.37\% images from iPohone-

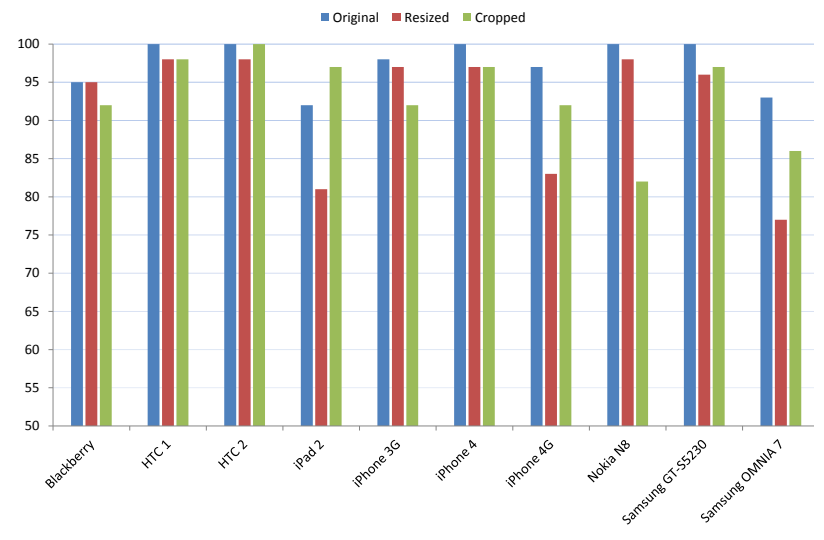

Fig. 4: Identification accuracy of individual devices using original images in training and modified images in testing.

4G were misclassified as originating from the iPad device and $5.56 \%$ images from Samsung GT were misclassified as Samsung Omnia 7 images. Similar results were achieved when training with original size images and testing with resized images. The overall accuracy was $72.2 \%$.

The experimental results indicate that higher identification rates can be achieved when modified images are compared against a training set of similar images (e.g. cropped vs. cropped) as opposed to original images (e.g. cropped vs original). However, as highlighted in [9], determining the type of modification or the transformation function applied to an image could be challenging.

\section{CONCLUSIONS}

We addressed the potential of using previously proposed image features to classify images from modern smartphones. We investigated the use of colour, image quality and wavelet features to identify the smartphone model of a given unknown image using a purpose built smartphone image database. Practically, it is possible to trace an image source model without prior knowledge of the model/device. We found that quality and wavelet features to be the most significant image features. Also, increasing the number of training images lead to improved identification accuracy.

The challenge of handling modified images was considered. In general, identification accuracy dropped significantly when the unknown image is a modified version of an original image. Higher identification rates can be achieved when modified images are tested against a modified training dataset as opposed to original dataset. Increasing the number of smartphone devices and adding other significant features that will increase the robustness of the developed model will be the focus of our future work. Furthermore, we hope to investigate the use of genetic algorithms for feature selection and feature weighting at feature-level and decision-level fusion. 


\section{REFERENCES}

[1] Sz-Han Chen and Chiou-Ting Hsu, "Source camera identification based on camera gain histogram," September 2007, Proc. IEEE Intl. Conf. Image Processing, pp. IV-429-IV-432.

[2] K.S. Choi, E.Y. Lam, and K.K.Y. Wong, "Source camera identification by jpeg compression statistics for image forensics," in TENCON 2006. 2006 IEEE Region 10 Conference, November 2006, pp. 1-4.

[3] Yongjian Hu, Chang-Tsun Li, and Changhui Zhou, "Selecting forensic features for robust source camera identification," in Proc. Intl. Computer Symposium. (ICS), 2010, pp. 506-511.

[4] J. Lukas, J. Fridrich, and M. Goljan, "Digital camera identification from sensor pattern noise," IEEE Trans. on Information Forensics and Security, vol. 1, no. 2, pp. 205-214, June 2006.

[5] M. Kharrazi, H.T. Sencar, and N Memon, "Blind source camera identification," October 2004, Proc. Intl. Conf. Image Processing, pp. 709-712.

[6] O. Celiktutan, B. Sankur, and I Avcibas, "Blind identification of source cell-phone model," IEEE Trans. on Information Forensics and Security, vol. 3, no. 3, pp. 553-566, September 2008.

[7] Min-Jen Tsai, Cheng-Liang Lai, and Jung Liu, "Camera/mobile phone source identification for digital forensics," in Acoustics, Speech and Signal Processing, 2007. IEEE International Conference on, April 2007, vol. 2 of Proc. ICASSP 2007, pp. II-221 -II-224.

[8] Thomas Gloe, Karsten Borowka, and Antje Winkler, "Information hiding," chapter Feature-Based Camera Model Identification Works in Practice, pp. 262-276. Springer-Verlag, Berlin, Heidelberg, 2009.

[9] Thomas Gloe and Rainer Bohme, "The dresden image database for benchmarking digital image forensics," Journal of Digital Forensic Practice, vol. 3, no. 2-4, pp. 150-159, 2010.

[10] Lanh Tran Van, S. Emmanuel, and M.S. Kankanhalli, "Identifying source cell phone using chromatic aberration," in Proc. IEEE Int'. Multimedia and Expo Conf, 2007, pp. 883-886.

[11] Chih-Chung Chang and Chih-Jen Lin, "Libsvm: A library for support vector machines," ACM Trans. Intell. Syst. Technol., vol. 2, no. 3, pp. 27:1-27:27, May 2011. 